\title{
A Conformal, Dynamic Pattern-Reconfigurable Antenna Using Conductive Textile-Polymer Composite
}

\author{
Bahare Mohamadzade, Student Member, IEEE, Roy B. V. B. Simorangkir, Member, IEEE, Raheel M. \\ Hashmi, Member, IEEE, Reza Gharaei, Ali Lalbakhsh, Member, IEEE, Sujan Shrestha, Student Member, IEEE, \\ Maxim Zhadobov, Senior Member, IEEE, and Ronan Sauleau, Fellow, IEEE
}

\begin{abstract}
A conformal antenna with electronically tuning capability of its radiation pattern between broadside and monopolelike patterns is proposed. The antenna is based on a proximityfed circular patch, loaded with a ring patch and four rectangular slots. The design is planar without any use of rigid shorting posts or complex feeding network. The reconfigurability is achieved by activating and deactivating the slots using PIN diodes, to switch between $\mathbf{T M}_{02}$ (monopole-like mode) and perturbed $\mathbf{T M}_{02}$ distributions (broadside mode) of the antenna. For conformability, the antenna is fabricated using highly flexible PDMSconductive fabric composite. All the antenna parts, including the RF switches, wires, and DC biasing circuit are fully encapsulated by PDMS to provide resilience against deformation and harsh environment. Investigations on the RF performance and mechanical stability of the antenna were conducted. Under various bendings, it was demonstrated that all the antenna components, including those for electronic switching, remained intact and in working order even under radius bending of $30 \mathrm{~mm}$, thus maintaining good pattern reconfigurability and overall performance. When bent, the measured results at $5.2 \mathrm{GHz}$ show a stable radiation performance relative to those of the flat case (i.e., maximum gain of $2.9 \mathrm{dBi}$ and efficiency of $64 \%$ in broadside mode, corresponding to $1.75 \mathrm{dBi}$ and $52 \%$ in monopole-like mode). To the best of our knowledge, all these features have never been demonstrated in previously published pattern reconfigurable antennas.
\end{abstract}

Index Terms-Conductive textile, conformal antenna, conformal antenna, pattern reconfigurable antenna, polymer.

\section{INTRODUCTION}

With the rapid development of modern wireless communication systems, antennas with pattern diversity have drawn a lot of attention. These include antennas that can switch dynamically their radiation pattern between broadside and monopole-like patterns. Switching radiation patterns enhances wireless system performance through its potential to avoid interference with noise sources, to provide wider coverage and

This work was supported in part by the International Macquarie University Research Excellence Scholarship (iMQRES) by the Australia Research Council.

B. Mohamadzade, R. M. Hashmi, S. Shrestha and Ali Lalbakhsh are with the School of Engineering, Macquarie University, Sydney, NSW 2109, Australia (email: bahare.mohamadzade@students.mq.edu.au).

R. B. V. B. Simorangkir is with Tyndall National Institute, University College Cork, Dyke Parade, T12R5CP Cork, Ireland.

M. Zhadobov and R. Sauleau are with the University of Rennes 1, CNRS, Institut d'Electronique et de Télécommunications of Rennes (IETR)-UMR 6164, F-35000 Rennes, France (e-mail: maxim.zhadobov@univ-rennes1.fr).

R. Gharaei is with the Faculty of Engineering, Islamic Azad UniversitySouth Tehran Branch, Tehran 15847-43311, Iran. to save energy [1]-[3]. This makes such antennas attractive for various applications, including cognitive radio [4], [5], indoor wireless network [1], [6]-[8], base station [9], wireless body area network (WBAN) [10]-[14], and multi-input multioutput (MIMO) system [3], [8], [13]. In the modern wireless communication systems, there is also a demand for pattern reconfigurable antennas that are conformal or bendable. The revolutionized concept of 5G and Internet of Things (IoT) invokes the need for high performance integrated networks, which imposes infrastructure challenges especially in urban area as well as the users' compliance. As compared to the rigid reconfigurable antennas, their conformal counterparts would benefit from their flexible deployment on the system platforms regardless the shape of the surface, optimum use of limited spaces or existing infrastructure, as well as the users' unobtrusiveness and comfort. In addition, having a simple method of controlling antenna's pattern and integrating the whole of controlling system with the antenna is an important advantage of a pattern reconfigurable antenna.

Many antennas with pattern reconfiguration between broadside and monopole-like have been proposed. Usually this is achieved by reconfiguring different operating modes of the antenna, each yielding distinctive radiation characteristic. This is made possible by incorporating RF switches with the radiating elements [1], [3], [10]-[20] or in the feeding network [7], [8], [21]-[26]. For instance, in [1], [3], [11], [12], [15], [19], [20], PIN diodes were used to reactively load the antenna with shorting posts/walls, switching between broadside mode and monopolar mode. A different approach to achieve broadside-monopolar pattern reconfigurability was demonstrated in [6], [24], [27]-[36]. That was by adopting two- or three-port excitation system for either single or multiple radiating elements. This also allows a continuous null steering by simultaneously feeding the antenna through two ports, with proper power ratio and phase delay between the two ports [27], [28]. Another unique way to continuously steer the antenna null direction was reported in [4]. Four varactortuned parasitic patches loaded at the perimeter of a center-fed cross-shaped patch, were used to imbalance the monopolar current distribution of the cross-shaped patch, hence yielding a continuous tilt in the antenna pattern.

In previous works, broadside-monopolar pattern reconfiguration requires a feeding network, which is often complex. At times, the need for several RF switches and shorting posts also 


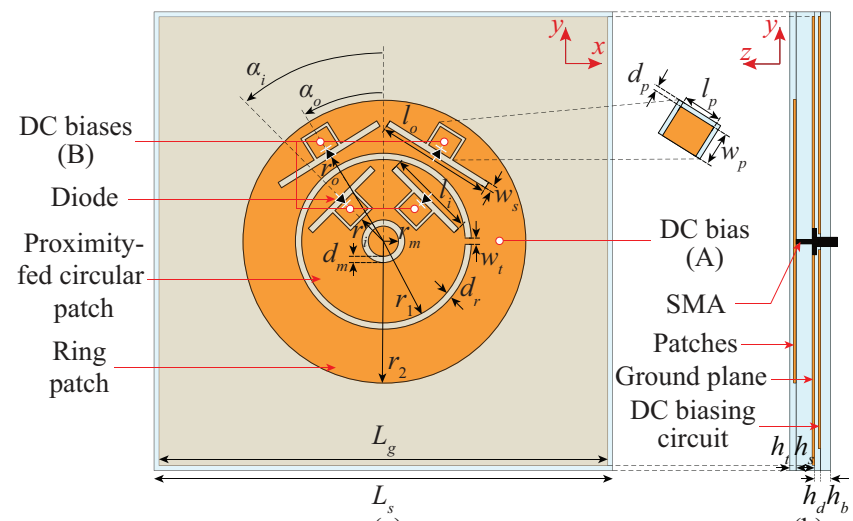

(a)

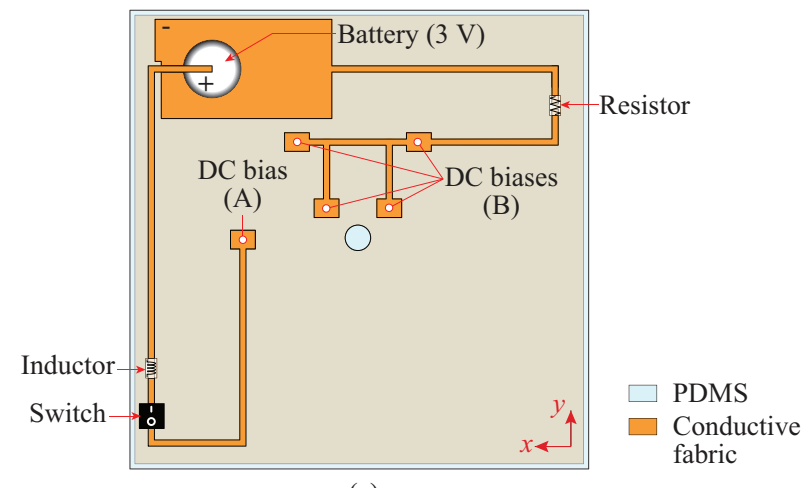

(c)

Fig. 1. Proposed antenna geometry: (a) front view, (b) side view, and (c) back view. The final dimensions (in millimeters) are: $r_{m}=2.4, d_{m}=1, r_{1}=13$, $r_{2}=22.5, d_{r}=1, l_{i}=14, l_{o}=18.5, w_{s}=1, r_{i}=4.9, r_{o}=15.8, \alpha_{i}=45^{\circ}$, $\alpha_{o}=32^{\circ}, l_{p}=4, w_{p}=3.5, d_{p}=0.5, w_{t}=1, L_{s}=70, L_{g}=68, h_{t}=0.3$, $h_{s}=5.5, h_{d}=0.3$, and $h_{b}=0.5$

increases the complexity of the antenna design process and fabrication. In addition, all of the presented works are based on rigid structures. To the best of our knowledge, there have been very limited attempts reported on flexible antenna, as opposed to their counterparts with rigid materials [37]. There was indeed an interesting work based on textile materials reported in [11], which did show a potential to have broadside and monopole-like patterns switching on flexible substrate. However, the electronic tuning of the antenna with actual switches have not been demonstrated yet.

In this paper, we present for the first time a conformal antenna that can switch electronically its radiation characteristic between broadside and monopole-like patterns. Unlike most of previously reported work, this proposed proximity-fed circular patch antenna is completely planar in structure, without any use of rigid shorting posts and complex feeding network, which facilitates very well its realization on flexible materials. The design also only utilizes 4 PIN diodes with a relatively simple DC biasing circuit design. We further employed flexible PDMS-conductive fabric composite material [38], [39] to realize the antenna prototype, in which all the antenna parts, including the DC biasing circuit, RF switches, wires, and other components are embedded inside PDMS, maintaining their integration to the flexible fabric. Therefore, while being conformal, the antenna is resilient against deformation and harsh environment, shown by good performance including reconfigurability, under different conformal scenarios.
The details of the antenna design and fabrication processes are given in the next section of the paper. It is followed by thorough investigations on the antenna performance in flat condition and under deformation.

\section{Antenna Design And Prototype}

\section{A. Antenna Configuration}

Fig. 1 shows the configuration of the proposed reconfigurable antenna. The antenna is based on a proximity-fed circular patch, loaded with a ring patch. The proximity feeding approach was used to give a wide bandwidth [40]-[43] and the circular proximity-fed structure was chosen to maintain the symmetry of the circular patch current distribution. For reconfigurability, four slots, each bridged to a rectangular pad by a PIN diode, are loaded to the patches. The radiators are all placed on a PDMS substrate having a rectangular-shaped ground plane on its opposite side. A thicker substrate was used to achieve more than 50\% radiation efficiency in both modes. Another solution that can be applied to improve the antenna efficiency would be the reduction of materials losses e.g., through mixing PDMS with micro/nanoparticle [44], [45] or repetitive coating of the conductive fabric [46]. Underneath the ground plane, separated by a thin PDMS layer, there is DC biasing circuit of the antenna. To provide physical robustness to the antenna, extra PDMS layers are added that completely encapsulate the antenna, including the DC biasing circuit and the lumped electronic components [38], [39], [47].

In simulations, a permittivity of 2.76 and increasing loss tangent from 0.03 to 0.06 , obtained through the Agilent 85070E Dielectric Probe Kit measurements from 3 to $7 \mathrm{GHz}$, were used to electrically model the PDMS material. On the other hand, the conductive parts of the antenna were modeled as a section having $0.08 \mathrm{~mm}$ thickness, which is the thickness of the conductive fabric used in the antenna realization (i.e., nickel-copper coated ripstop from Less EMF Inc.). The effective conductivity of $5.4 \times 10^{4} \mathrm{~S} / \mathrm{m}$, was used for PDMSconductive fabric composite, considering the percolation of the PDMS into the pores of the chosen fabric during the integration of both materials [39], [47].

Four SMP1345-079LF PIN diodes from Skyworks [12], [48] were used in the antenna design. The cathode of each diode is connected to the rectangular pad, while its anode is connected to the antenna patch. The ring and circular patches are connected to each other with a very small strip to provide a same DC plane without affecting the RF performance of the antenna. The DC biasing circuit underneath the ground is connected to the rings and the rectangular pads at points A (positive polarity) and B (negative polarity), respectively, by means of thin wires piercing through the PDMS. The insulation of the wires isolate the pads and the rings from the RF ground. As the DC voltage supply, a $3 \mathrm{~V}$ coin cell battery is used, which is connected to a MCDHN-02F-V dip switch from Multicomp Pro to control the status of the diodes. A $100 \mathrm{nH}$ chip inductor (0805CS-060XJLB) from Coilcraft is used as an RF choke, while on the other hand, a $82 \Omega$ surface mount resistor (MC 0805) from Multicomp Pro is used to limit the current passing through each diode.

\section{ACCEPTED MANUSCRIPT}




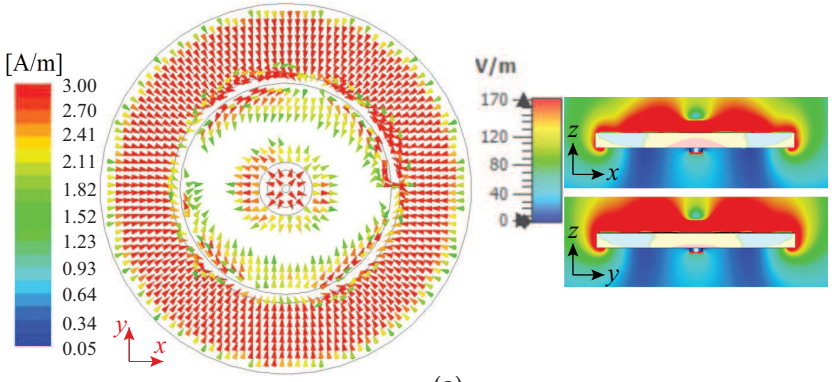

(a)
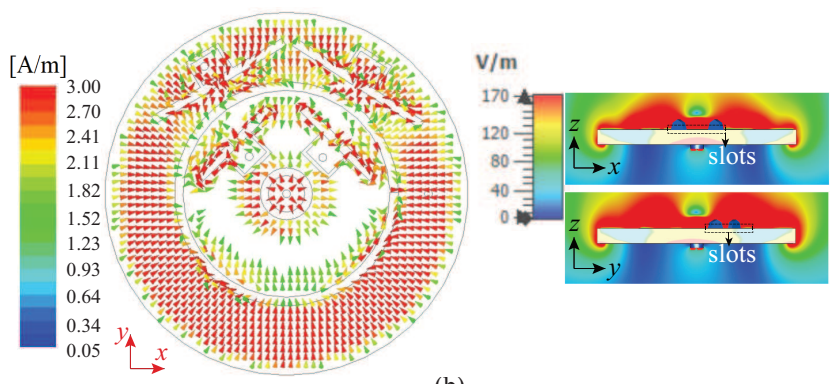

(b)
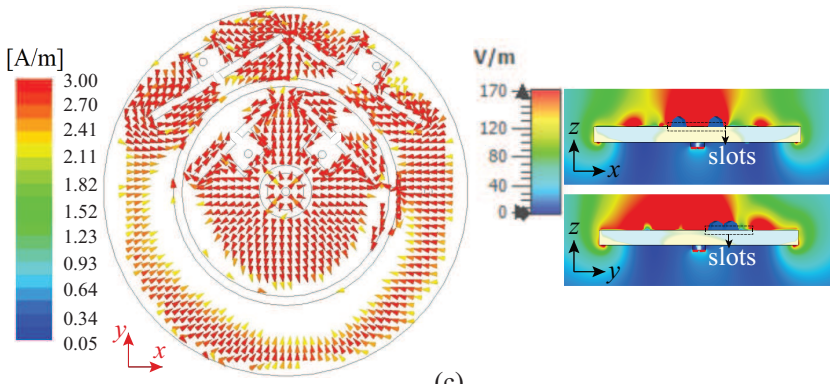

(c)

Fig. 2. Simulated current (left) and average E-field (right) distributions at $5.2 \mathrm{GHz}$ of the proposed antenna: (a) without slots, (b) with slots and diodes $\mathrm{ON}$, (b) with slots and diodes OFF.

TABLE I

VARIATION OF Slot PARAMETERs to Illustrate Monopolar to BROAdSIDE PATTERN TUNING

\begin{tabular}{c|l}
\hline Par. Set & Dimensions $(\mathrm{mm})$ \\
\hline \hline Set 1 & $l_{i}=12, l_{o}=9, \alpha_{i}=45^{\circ}, \alpha_{o}=45^{\circ}, r_{i}=4.1, r_{o}=16$ \\
Set 2 & $l_{i}=10, l_{o}=13, \alpha_{i}=60^{\circ}, \alpha_{o}=38^{\circ}, r_{i}=5.4, r_{o}=15.4$ \\
Set 3 & $l_{i}=11, l_{o}=14, \alpha_{i}=80^{\circ}, \alpha_{o}=32^{\circ}, r_{i}=5.8, r_{o}=16.3$ \\
Set 4 & $l_{i}=14, l_{o}=14, \alpha_{i}=50^{\circ}, \alpha_{o}=40^{\circ}, r_{i}=4.5, r_{o}=15$ \\
Set 5 & $l_{i}=14, l_{o}=18.5, \alpha_{i}=45^{\circ}, \alpha_{o}=32^{\circ}, r_{i}=4.9, r_{o}=15.8$ \\
\hline
\end{tabular}

\section{B. Pattern Reconfigurability Mechanism}

The proximity-fed circular patch antenna was designed to operate in its monopolar operating mode. In this work, the $\mathrm{TM}_{02}$ operating mode was chosen for its well-known monopole-like radiation pattern [49] with better quality of gain and azimuthal omnidirectional pattern [50]. The ring was loaded at the edge the circular patch to shift down the $\mathrm{TM}_{02}$ resonance closer to the target frequency of $5.2 \mathrm{GHz}$. This was made possibly by a proper coupling between the ring and circular patches, obtained through an optimization of the radius of the ring and its gap to the circular patch [51].

The idea behind the slots inclusion on the antenna patches is to disturb the balance of the $\mathrm{TM}_{02}$ radial current distribu- tion. Based on a feasibility study that we conducted on the effect of the number for slots (not shown here for brevity), four slots are chosen considering its effectiveness as well as design simplicity. Each pair of slots is positioned in both inner and outer patches, respectively, to maximize the current disturbance. From simulations, we found that with proper disturbance, achieved through an optimization of the slot dimensions (i.e., $l_{i}, l_{o}$ ) and positions (i.e., $r_{i}, r_{o}, \alpha_{i}, \alpha_{o}$ ), the null direction of the $\mathrm{TM}_{02}$ monopole-like pattern can be shifted from its broadside direction. As a result, transformation from a monopole-like to normal patch mode pattern is achieved. PIN diodes are used to activate and deactivate the slots, which allows for switching between the two radiation modes. This is the strategy to achieve the pattern reconfigurability feature of the proposed antenna.

The current distribution of the antenna are plotted in Fig. 2, along with the respective E-field distributions. As shown in Fig. 2(b), when the slots are deactivated (diodes ON), there is minimum current diversion as most of the current can pass through the middle of the slots and the rectangular pads. As a result, similar current distribution and E-field, to the case before the slots inclusion, are obtained (compare Fig. 2(b) and (a)). On the other hand, as shown in Fig. 2(c), when the slots are activated (diodes OFF), the current must flow around the slots, which yields a shift of the E-field null from the center of the patches, towards the location of the slots where the current is disturbed.

To illustrate further the role of the slots in the antenna pattern modification, the antenna was simulated for different sets of parameters given in Table I and the total radiation patterns are plotted in Fig. 3. It should be noted that this parametric study was conducted when all diodes were in OFF state, with all other parameters set to constant as given in the caption of Fig. 1. We found that to achieve the desired pattern transformation, the slots optimization should aim for: (1) maximizing the current diversion around the slots, and (2) concentrating the disturbance of the current at one of the poles of the patches. For the latter, it is important to have both inner and outer pair of slots placed at the same pole of the patches and are positioned close to the gap between the inner and outer patches $\left(d_{r}\right)$. As we were limited by the space required on the patches for DC bias pads when tuning the slots radial positions $\left(r_{i}\right.$ and $\left.r_{o}\right)$, we observed that the lengths $\left(l_{i}\right.$ and, $\left.l_{o}\right)$ and the rotation angles $\left(\alpha_{i}\right.$ and $\left.\alpha_{o}\right)$ of the slots have major role in shifting the antenna pattern. It is depicted in Fig. 3 that the shift in the E-field null shown in Fig. 2(c) can also be seen as the monopolar pattern being slanted towards the location of the slots or positive theta direction as the dimensions of the slots are optimized. Once the null of the monopolar pattern is slanted further from the broadside direction, a patch-like pattern is produced. It is implied from our investigation (see Fig. 2(c) and Fig. 3) that the pattern symmetricity especially in the yz-plane could be improved by shifting further the E-field null from the center so that the peak of the half of the monopolar pattern is fully translated to the zero-theta direction (see black circle on the patterns in Fig. 3). This is possible through a more complex design and arrangement of the slots. Nevertheless, looking

\section{ACCEPTED MANUSCRIPT}




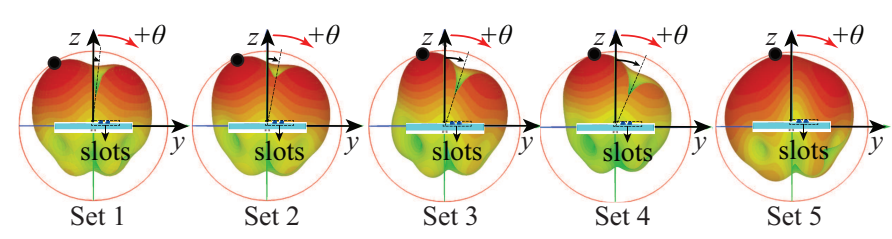

Fig. 3. Simulated total radiation pattern of the antenna at $5.2 \mathrm{GHz}$ when the diodes are OFF, with varying slot parameters.

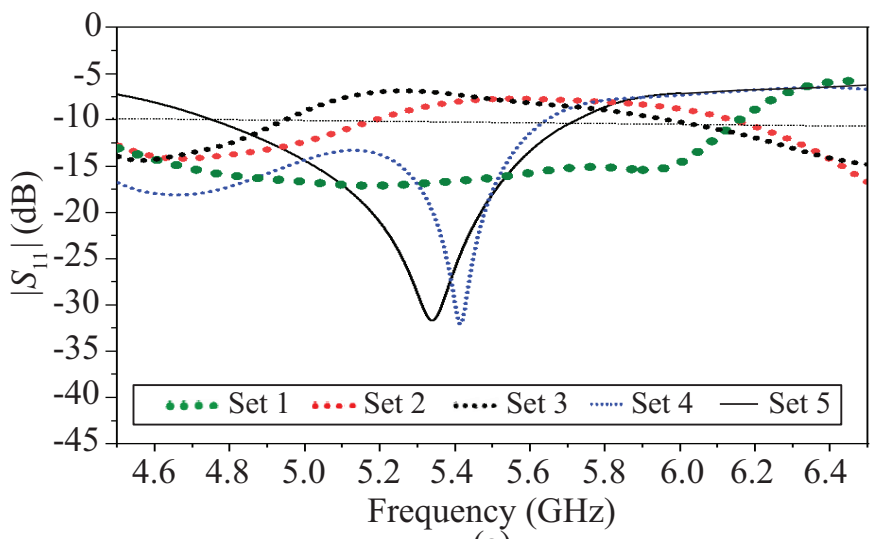

(a)

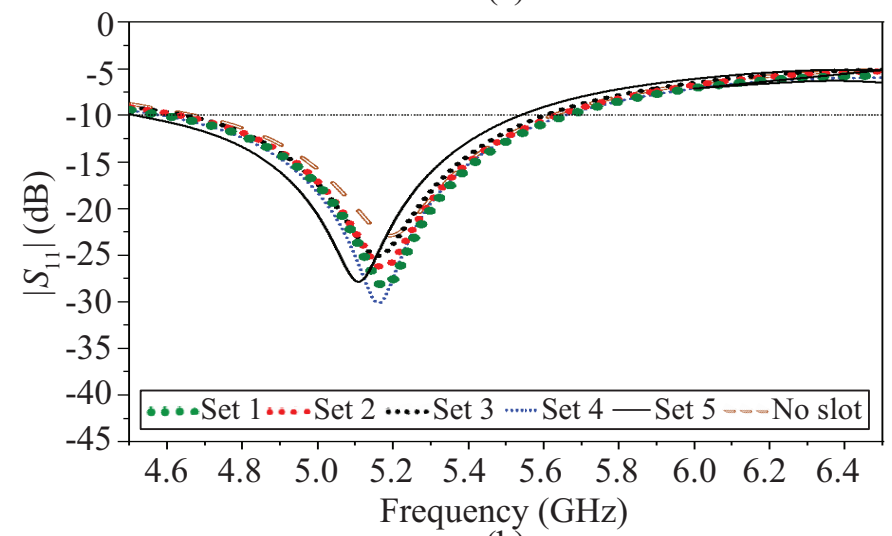

(b)

Fig. 4. Simulated $\left|S_{11}\right|$ of the antenna when the slot parameters are varied for (a) diode $\mathrm{OFF}$ and, (b) diodes $\mathrm{ON}$

at the direction of the main lobe, the antenna still fulfills very well the broadside radiation characteristic that is aimed for. The reflection coefficient $\left|S_{11}\right|$ corresponding to the slot variations is shown in Fig.4, which shows how the antenna matching being affected by the slot modifications. Therefore, a thorough optimization of the antenna dimensions, particularly the slot-related parameters, was conducted with an aim to achieve a broadside radiation pattern and good matching at the targeted operating frequency, while taking into consideration the fabrication tolerance.

\section{Antenna Prototyping}

As a proof of concept, the proposed design was fabricated through a layer-by-layer assembly process of PDMSconductive fabric composite introduced in [38], [39]. Several rectangular ring-shaped molds with corresponding thicknesses were used to achieve the specified PDMS layers. The conductive sections were cut out of the conductive fabric by hand

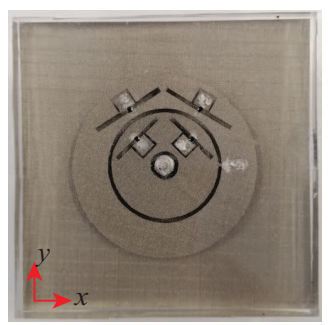

(a)

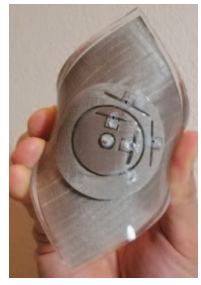

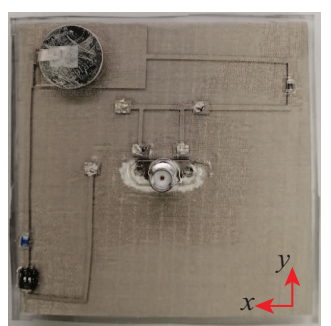

(b)

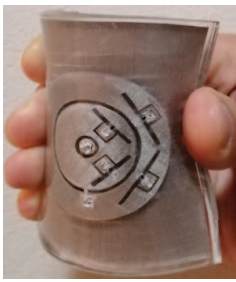

(c)

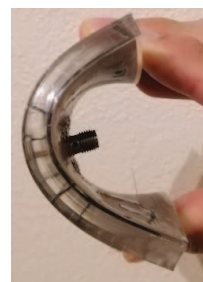

Fig. 5. Prototype of the proposed reconfigurable antenna: (a) top view, (b) bottom view, and (c) view when deformed under various bendings demonstrating its conformability.

using a razor blade. The fabrication was done in bottomup approach, starting from the bottom encapsulation layer, followed by the DC biasing circuit, thin separator layer, ground plane, PDMS substrate, antenna patches, and finally the upper encapsulation layer. The PIN diodes were attached to the pads and patches before layering the upper PDMS encapsulation. On the other hand, other components (e.g., the inductor, resistor, dip switch, battery, and SMA connector) and wires were connected after taking out the cured prototype from the mold. To connect these components, parts of the PDMS encapsulation layers were scratched out slightly with razor blade. Upon the attachments, uncured PDMS was poured to fully cover these components, except for the battery, the SMA port, and the slide of the switch, for practicality of the measurements. Adhesive tape was used to maintain the battery connection with the conductive fabric used as the positive and negative leads.

The attachment of the conductive fabric on the cured PDMS layers was done by using uncured PDMS, while the attachment of the electronic components and wires to the conductive fabric was done by means of silver epoxy. For the latter, it is important to maintain an efficient use of the epoxy to minimize the area of fabric that become less flexible upon the curing of the epoxy [52].

Fig. 5 shows the photographs of the fabricated prototype. The prototype robustness against deformation was tested initially through various concave bending tests with hands as illustrated in Fig. 5(c), which was verified further through experiments in next section. Owing to the flexibility of the composite material, the antenna can be bent without breaking. It was noticed that visually, under such level of bending (average radius of $30 \mathrm{~mm}$ ), the integration of the lumped components on the body of the antenna was also maintained, demonstrating conformability of the proposed reconfigurable antenna. However, it was observed that the slot widths started to expand, giving more pressures to the fabric and diodes interconnections. This was likely caused by the concave bending

\section{ACCEPTED MANUSCRIPT}




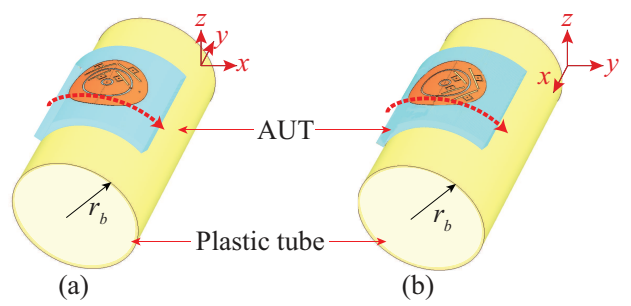

Fig. 6. Illustration of the antenna conformed around the plastic tube during $S_{11}$ and far-field measurements: (a) along $x$-axis direction, (b) along $y$-axis direction. (The axes are respected to the antenna)

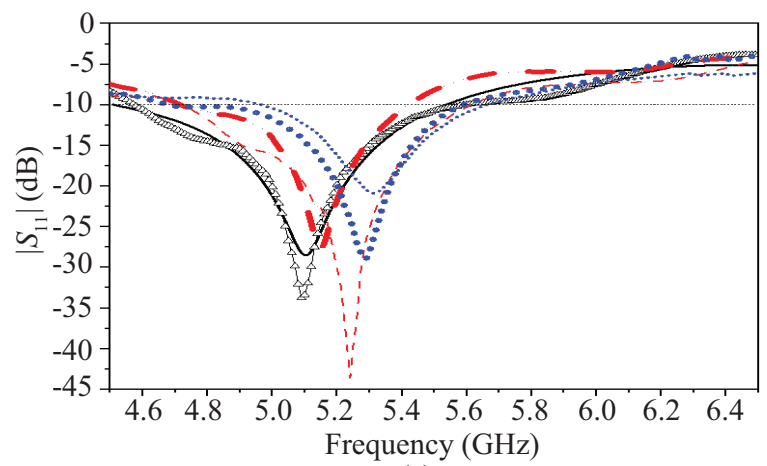

(a)

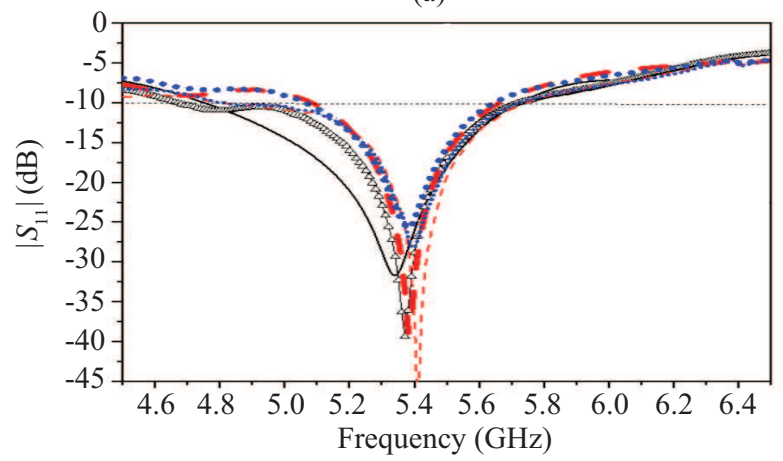

(b)

\begin{tabular}{|c|c|}
\hline $\begin{array}{l}\text { - Sim. flat } \\
-- \text { Meas. } r_{b}=40 \mathrm{~mm} x \text {-axis } \\
-- \text { Meas. } r_{b}=40 \mathrm{~mm} y \text {-axis }\end{array}$ & $\begin{array}{l}\because \text { Meas. flat } \\
\cdots \cdots \cdots \text { Meas. } r_{b}= \\
\cdots \cdots \text { Meas. } r_{b}=\end{array}$ \\
\hline
\end{tabular}

Fig. 7. $\left|S_{11}\right|$ performance of the proposed antenna in both flat and conformed conditions: (a) when all diodes ON and (b) when all diodes OFF. For the conformed case, the results of two different bending radii, in both $x$ - and $y$-axis directions, are shown.

configuration and the elastic nature of the PDMS to which the fabric is attached. On the other hand, it was also observed that repetitive bendings with such small radius seemed to affect the conductive fabric and SMA connector interconnection, possibly due to the size of the connector that is comparable to the antenna bending curvature.

\section{RESUlts}

The antenna performance was measured under flat condition and under deformation, to confirm its conformability and mechanical robustness. Hollow plastic tubes having an outer radius of $r_{b}$ were used in deformation case. The antenna was concavely conformed over the tubes by means of adhesive tape, in two different configurations, i.e., along $x$ - and $y$ axis directions (see Fig. 6). Underneath the antenna, $3 \mathrm{~mm}$
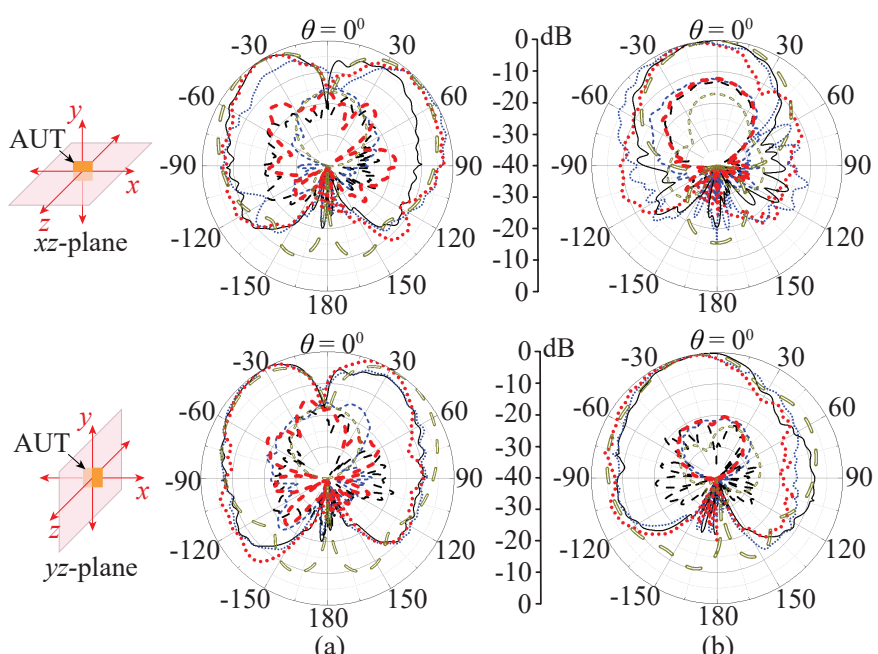

(a)

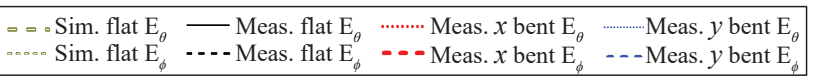

Fig. 8. Normalized simulated and measured radiation patterns of the proposed antenna in flat and bent $\left(r_{b}=40 \mathrm{~mm}\right)$ conditions: (a) when all diodes ON and (b) when all diodes OFF

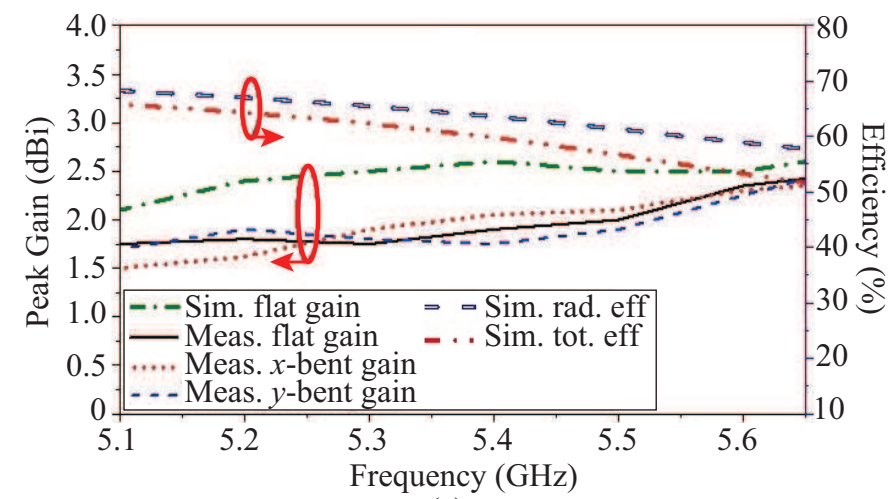

(a)

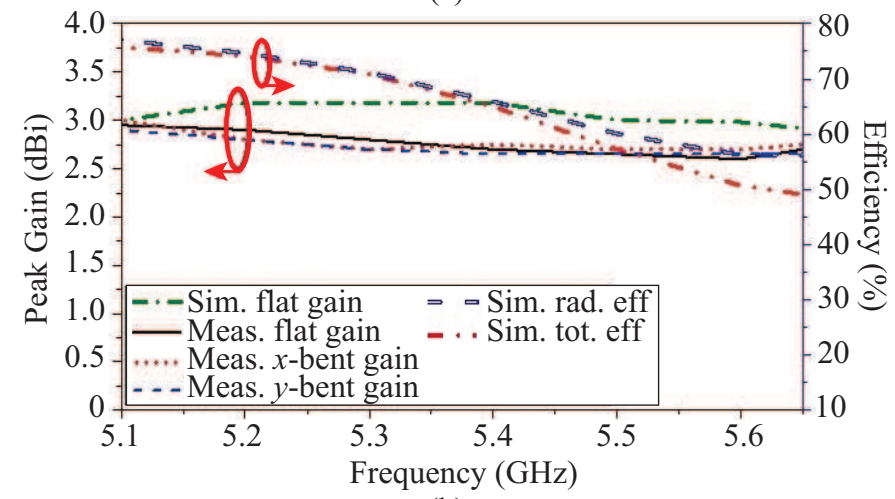

(b)

Fig. 9. Antenna peak gains in both flat and conformed $\left(r_{b}=40 \mathrm{~mm}\right)$ conditions: (a) when all diodes ON and (b) when all diodes OFF

thickness foam was used to facilitate the gap created by the battery. A hole was made on the tubes to facilitate the coaxial cable for the measurements. The input reflection coefficient $\left(\left|S_{11}\right|\right)$ was measured with Agilent PNA-X N5242A network analyzer, while the antenna radiation characteristics were measured in the NSI700S-50 spherical near-field antenna range at the Australian Antenna Measurement Facility (AusAMF). 
TABLE II

Summary of The Antenna Measured Bandwidth and Peak Gain (AT 5.2 GHz) UNDER FLAT AND BENDING CONDITIONS

\begin{tabular}{l|c|c|c|c}
\hline \multirow{2}{*}{\multicolumn{1}{c|}{ Condition }} & \multicolumn{2}{|c|}{ Bandwidth $(\mathrm{GHz})$} & \multicolumn{2}{c}{ Peak Gain (dBi) } \\
\cline { 2 - 5 } & Monopole & Broadside & Monopole & Broadside \\
\hline \hline Meas. flat & $4.57-5.59$ & $4.67-5.68$ & 1.75 & 2.90 \\
\hline Meas. $x$-axis bent: & & & & \\
$r_{b}=30 \mathrm{~mm}$ & $5.05-5.60$ & $4.88-5.72$ & - & - \\
$r_{b}=40 \mathrm{~mm}$ & $4.72-5.62$ & $4.89-5.74$ & 1.60 & 2.60 \\
\hline Meas. $y$-axis bent: & & & & \\
$r_{b}=30 \mathrm{~mm}$ & $4.72-5.50$ & $5.10-5.65$ & - & - \\
$r_{b}=40 \mathrm{~mm}$ & $4.72-5.41$ & $5.12-5.64$ & 1.85 & 2.80 \\
\hline
\end{tabular}

\section{A. Input Reflection Coefficient}

The $\left|S_{11}\right|$ results of the antenna under flat and various bendings, for both states, are shown in Fig. 7. In flat case, the measured results are shown together with the results obtained from numerical simulations, which are in a good agreement. Both results show a slight shift in the antenna resonance frequency upon the change of the diodes state. Nevertheless, the targeted frequency of $5.2 \mathrm{GHz}$ is still covered within the 10-dB return loss bandwidth of both states. The measured overlapping bandwidth in which the antenna could excite both modes is from 4.67 to $5.59 \mathrm{GHz}(17.9 \%)$. There are indeed slight differences between the measured and simulated results, which are most likely attributed to the inaccuracies in minute fabrication (e.g., during the manual cutting of the conductive fabric, the arrangement of the fabric cuts on the PDMS surface, and the assembly of the DC biasing circuit and components) or modeling of the diode.

When the antenna was bent around two different tubes, $r_{b}=40$ and $30 \mathrm{~mm}$, the antenna was still found operational. There are some variations in the $\left|S_{11}\right|$ under bendings, which are expected due to the changes in the current paths upon the change of the physical structure of the antenna.

\section{B. Radiation Performance}

The simulated and measured radiation patterns of the antenna at $5.2 \mathrm{GHz}$ for flat and bent cases are shown in Fig. 8 . For the far-field measurements of the antenna under bending, only the tube with $r_{b}=40 \mathrm{~mm}$ was used. The measured results of the unbent antenna clearly show that the antenna radiation characteristic change upon activating and deactivating the diodes, which agrees very well with the simulated results. When all the diodes are $\mathrm{ON}$, the antenna radiation resembles that of a vertical monopole antenna, but when the diodes are OFF, the antenna radiates in broadside manner. Under bendings, the patterns are slightly deviated (e.g., the beam width, the cross-polarization level, and the null position), which can be understood as the effect of the structural deformation of the antenna. However, the most important point to highlight is that the antenna reconfigurability feature between monopolelike and broadside patterns is well retained. This particularly confirms that the integration of the rigid lumped elements used for electronic tuning on the flexible body of the antenna, are indeed maintained even under deformation, demonstrating the resilience of the antenna to physical deformation.

Fig. 9 provides the information of the antenna peak gains as a function of frequency, shown in both states, under flat and bent conditions. The measured results of the unbent antenna at $5.2 \mathrm{GHz}$ show maximum gains of 2.9 and $1.75 \mathrm{dBi}$ with $64 \%$ and $52 \%$ radiation efficiencies, in broadside and monopolelike modes, respectively with current ground plane $\left(1.2 \lambda_{0}\right.$ $\times 1.2 \lambda_{0}$ ). Higher gain can be achieved by having bigger ground plane. Slight discrepancies from the simulated results were noticed, which are attributed to the minor variations in fabrication process. One of the parameters that may affect the measured gain is the thickness of top layer PDMS. Higher thickness reduces the gain of antenna. This is because of the loss in the PDMS itself. Moreover, more differences in the monopole-like mode can be as a result of the diode modelling which is described before. Under $40 \mathrm{~mm}$ bending radius in both $x$ - and $y$-axis cases, the antenna gain performance is seen to be relatively stable. Lastly, the summary of the antenna performance in different antenna conditions is provided in Table II.

\section{Discussion}

Table III summarizes the properties of the proposed antenna in comparison with the other reported broadside and monopolar patterns reconfigurable antennas. Referring to these tabulated results, only our antenna and antenna reported in [11] are conformal whereas in our antenna, actual diodes were employed to switch between the two radiation modes. While being the only conformal pattern reconfigurable antenna, our design still demonstrates good performance. As compared to other proposed antennas, the design is also simpler with relatively moderate electrical size, thickness, as well as the number of diodes used. There are no shorting pins/walls or complex feeding network utilized in the proposed design. The DC biasing part is relatively simple and integrated at the back of the antenna, eliminating the dangling cables usually associated with the bias network. Therefore, the proposed antenna with its ability for switching between broadside pattern and monopole-like pattern at $5.2 \mathrm{GHz}$ is a promising candidate for applications in WLAN and short-range wireless sensor networks within a limited area such as a home, school, computer laboratory, campus, office building, vehicle, etc. Its flexibility allows it to be placed on curved surfaces, such as autonomous terrestrial vehicles without any difficulties that rigid structures have.

\section{CONCLuSion}

We have successfully demonstrated for the first time a conformal reconfigurable antenna for dynamic switching between monopole-like and broadside patterns. The reconfigurability was achieved by the inclusion of four slots, controlled by PIN diodes, on a proximity-fed circular patch, to switch between its $\mathrm{TM}_{02}$ (monopole-like mode) and perturbed $\mathrm{TM}_{02}$ distributions (broadside mode). These characteristics were achieved without using any vertical vias, matching networks or multiple feeding points. The planar structure of the proposed design makes

\section{ACCEPTED MANUSCRIPT}


TABLE III

COMParison with State-OF-THE-ARt Broadside-Monopolar Pattern Reconfigurable Antennas

\begin{tabular}{c|c|c|c|c|c|c|c|c|c}
\hline Ant. & FC $(\mathrm{GHz})$ & OFBW $(\%)$ & FP $\left(\lambda_{o}{ }^{2}\right)$ & $\mathrm{H}\left(\lambda_{o}\right)$ & $\mathrm{N}$ & $\mathrm{CL}$ & $\mathrm{PGB}(\mathrm{dBi})$ & PGM $(\mathrm{dBi})$ & FN/BC/CP \\
\hline \hline$[1]$ & 2.02 & 2.47 & $0.67 \times 0.67$ & 0.05 & 2 & No & 6.8 & 2.5 & No/Yes/Yes \\
\hline$[2]^{*}$ & 2.10 & 2.10 & $0.7 \times 0.7$ & 0.04 & 12 & No & 7.9 & 2.1 & Yes/No/No \\
\hline$[3]$ & 5.32 & 6.60 & $0.89 \times 0.89$ & 0.06 & 8 & No & 6.5 & 3.5 & No/No/Yes \\
\hline$[4]$ & 2.43 & 3.29 & $1.22 \times 1.22$ & 0.03 & 12 & No & NA & NA & No/Yes/No \\
\hline$[8]$ & 2.80 & 60.7 & $1.21 \times 1.21$ & 0.17 & 6 & No & 8.5 & 6.0 & Yes/Yes/Yes \\
\hline$[10]$ & 2.31 & 8.21 & $0.69 \times 0.65$ & 0.06 & 2 & No & 4.1 & 4.8 & No/Yes/Yes \\
\hline$[11]^{*}$ & 2.44 & 4.69 & $0.81 \times 0.81$ & 0.02 & 6 & Yes & 3.9 & 2.0 & No/No/Yes \\
\hline$[12]^{* *}$ & 2.45 & 1.02 & $\pi \times 0.2^{2}$ & 0.03 & 6 & No & 3.8 & -3.0 & No/Yes/Yes \\
\hline$[15]$ & 2.4 & 2.50 & $\pi \times 0.64^{2}$ & 0.04 & 16 & No & 6.05 & 4.39 & No/Yes/Yes \\
\hline$[21]$ & 2.55 & 7.84 & $1.15 \times 1.15$ & 0.12 & 12 & No & 7.5 & 5.8 & Yes/Yes/Yes \\
\hline$[24]$ & 1.85 & 24.3 & $\pi \times 1.23^{2}$ & 0.11 & 0 & No & 8.5 & 3.5 & Yes/No/Yes \\
\hline$[27]$ & 2.44 & 3.28 & $0.98 \times 0.81$ & 0.05 & 0 & No & 8.9 & 4.34 & Yes/No/Yes \\
\hline$[28]$ & 1.60 & 5.43 & $0.48 \times 0.48$ & 0.07 & 0 & No & NA & NA & No/No/Yes \\
\hline This work & 5.13 & 17.9 & $1.2 \times 1.2$ & 0.11 & 4 & Yes & 2.9 & 1.75 & No/Yes/No \\
\hline
\end{tabular}

Note: $\mathrm{FC}=$ center frequency of overlapping bandwidth between two modes $(\mathrm{OFBW}), \mathrm{FP}=$ overall footprint, $\mathrm{H}=$ overall thickness, $\lambda_{o}=$ free space wavelength of $\mathrm{FC}, \mathrm{N}=$ number of RF switches required (e.g., diode, varactor), $\mathrm{CL}=$ conformality, $\mathrm{PGB}=$ broadside peak gain at $\mathrm{FC}, \mathrm{PGM}=$ monopolar peak gain at $\mathrm{FC}, \mathrm{FN}=$ feeding network, $\mathrm{BC}=\mathrm{DC}$ biasing circuit, $\mathrm{CP}=$ multiple ports; shorting structures.

* Ideal stubs were used instead of RF switches. ** On-body performance only.

possible its realization using layer-by-layer assembly process of highly flexible PDMS-conductive fabric composite, in which all antenna elements are encapsulated inside the PDMS, including a relatively simple DC biasing circuit at the back of the antenna. This configuration provides physical robustness to the antenna makes it suitable for conformal modern wireless applications. The latter was validated experimentally through some mechanical tests, in which the antenna demonstrated that its reconfigurability was well preserved upon deformation, showing relatively stable impedance matching and gain performance.

\section{REFERENCES}

[1] S.-H. Chen, J.-S. Row, and K.-L. Wong, "Reconfigurable square-ring patch antenna with pattern diversity," IEEE Trans. Antennas Propag., vol. 55, no. 2, pp. 472-475, 2007.

[2] W. Cao, B. Zhang, A. Liu, T. Yu, D. Guo, and K. Pan, "A reconfigurable microstrip antenna with radiation pattern selectivity and polarization diversity," IEEE Antennas Wireless Propag. Lett., vol. 11, pp. 453-456, 2012.

[3] P. Qin, Y. J. Guo, A. R. Weily, and C. Liang, "A pattern reconfigurable U-slot antenna and its applications in MIMO systems," IEEE Trans. Antennas Propag., vol. 60, no. 2, pp. 516-528, 2012.

[4] S. Yong and J. T. Bernhard, "A pattern reconfigurable null scanning antenna," IEEE Trans. Antennas Propag., vol. 60, no. 10, pp. 45384544, 2012.

[5] S. Yong and J. T. Bernhard, "Reconfigurable null scanning antenna with three dimensional null steer," IEEE Trans. Antennas Propag., vol. 61, no. 3, pp. 1063-1070, 2013.

[6] L. Cui, W. Wu, and D. Fang, "Wideband circular patch antenna for pattern diversity application," IEEE Antennas Wireless Propag. Lett., vol. 14, pp. 1298-1301, 2015.
[7] W. Lin, H. Wong, and R. W. Ziolkowski, "Wideband patternreconfigurable antenna with switchable broadside and conical beams," IEEE Antennas Wireless Propag. Lett., vol. 16, pp. 2638-2641, 2017.

[8] X. Yang, H. Lin, H. Gu, L. Ge, and X. Zeng, "Broadband pattern diversity patch antenna with switchable feeding network," IEEE Access, vol. 6, pp. 69612-69619, 2018.

[9] J.-S. Row and Y.-J. Huang, "Reconfigurable antenna with switchable broadside and conical beams and switchable linear polarized patterns," IEEE Trans. Antennas Propag., vol. 66, no. 7, pp. 3752-3756, 2018.

[10] I. Lim and S. Lim, "Monopole-like and boresight pattern reconfigurable antenna," IEEE Trans. Antennas Propag., vol. 61, no. 12, pp. 5854-5859, 2013.

[11] S. Yan and G. A. Vandenbosch, "Radiation pattern-reconfigurable wearable antenna based on metamaterial structure," IEEE Antennas Wireless Propag. Lett., vol. 15, pp. 1715-1718, 2016.

[12] X. Tong, Ch. Liu, X. Liu, H. Guo, and X. Yang, "Switchable on/off body antenna for $2.45 \mathrm{GHz}$ WBAN applications," IEEE Trans. Antennas Propag., vol. 66, no. 2, pp. 967-971, 2017.

[13] J. Zhang, S. Yan, and G. A. E. Vandenbosch, "Realization of dual-band pattern diversity with a CRLH-TL-inspired reconfigurable metamaterial," IEEE Trans. Antennas Propag., vol. 66, no. 10, pp. 5130-5138, 2018.

[14] B. Mohamadzade, R. B. V. B. Simorangkir, R. M. Hashmi, and S. Shrestha, "Low-profile pattern reconfigurable antenna for Wireless Body Area Networks," in Proc. Int. Conf. on Electromag. in Adv. App. (ICEAA), 2019, pp. 546-547.

[15] Y. Yang, R. B. V. B. Simorangkir, X. Zhu, K. Esselle, and Q. Xue, "A novel boresight and conical pattern reconfigurable antenna with the diversity of $360^{\circ}$ polarization scanning," IEEE Trans. Antennas Propag., vol. 65 , no. 11 , pp. 5747-5756, 2017.

[16] Y.-Y. Bai, S. Xiao, C. Liu, X. Shuai, and B.-Z. Wang, "Design of pattern reconfigurable antennas based on a two-element dipole array model," IEEE Trans. Antennas Propag., vol. 61, no. 9, pp. 4867-4871, 2013.

[17] W. Kang, J. Park, and Y. J. Yoon, "Simple reconfigurable antenna with radiation pattern," Electronics Letters, vol. 44, no. 3, pp. 182-183, 2008.

[18] F. Sun, F. Zhang, and C. Feng, "A microstrip antenna for polarized 
diversity and pattern selectivity application," in Proc. Int. Conf. on Microw. and Millimeter Wave Tech. (ICMMT), 2018, pp. 1-4.

[19] N. Nguyen-Trong, L. T. Hall, and C. Fumeaux, "Impedance matching of a frequency and pattern-reconfigurable antenna," in Proc. $17^{\text {th }}$ Int. Symp. on Ant. Tech. and Applied Electromag. (ANTEM), 2016, pp. 1-2.

[20] $\mathrm{H}$. Li and S. He, "A compact reconfigurable antenna with pattern diversity," in Proc. IEEE Int. Symp. on Ant. and Propag. (ISAP), 2012, pp. $1-2$.

[21] W. Lin, H. Wong, and R. W. Ziolkowski, "Circularly polarized antenna with reconfigurable broadside and conical beams facilitated by a mode switchable feed network," IEEE Trans. Antennas Propag., vol. 66, no. 2, pp. 996-1001, 2018.

[22] W. Lin, R. W. Ziolkowski, and H. Wong, "Pattern reconfigurable techniques for LP and CP antennas with the broadside and conical beams," in Proc. 12 ${ }^{\text {th }}$ Eur. Conf. on Ant. and Propag. (EuCAP), 2018.

[23] X. Yang and L. Ge, "Broadband pattern diversity antenna with switchable feeding network," in Proc. Int. Conf. on Microw. and Millimeter Wave Tech. (ICMMT), 2019, pp. 1-3.

[24] S.-L. Yang and K.-M. Luk, "Design of a wide-band L-probe patch antenna for pattern reconfiguration or diversity applications," IEEE Trans. Antennas Propag., vol. 54, no. 2, pp. 433-438, 2006.

[25] H. Wong, W. Lin, X. Wang, and M. Lu, "LP and CP polarization reconfigurable antennas for modern wireless applications," in Proc. IEEE Int. Symp. on Ant. and Propag. (ISAP), 2017, pp. 1-2.

[26] J. Yang, W. Lin, and H. Wong, "Wideband CP polarization and pattern reconfigurable antennas," in Proc. IEEE Int. Conf. on Comp. Electromag. (ICCEM), 2018, pp. 1-2.

[27] X. Jiang, Z. Zhang, Y. Li and Z. Feng, "A novel null scanning antenna using even and odd modes of a shorted patch," IEEE Trans. Antennas Propag., vol. 62, no. 4, pp. 1903-1909, 2014.

[28] C. Deng, Y. Li, Z. Zhang, and Z. Feng, "A hemispherical 3-D null steering antenna for circular polarization," IEEE Antennas Wireless Propag. Lett., vol. 14, pp. 803-806, 2015.

[29] L. Sun, G.-x. Zhang, B.-h. Sun, W.-d. Tang, and J.-p. Yuan, "A single patch antenna with broadside and conical radiation patterns for $3 \mathrm{G} / 4 \mathrm{G}$ pattern diversity," IEEE Antennas Wireless Propag. Lett., vol. 15, pp. 433-436, 2015.

[30] Z. Pu and Z. Zhan, "A compact and wideband microstrip antenna design with switchable pattern between conical and broadside beam," in Proc. Int. Conf. on Microw. and Millimeter Wave Tech. (ICMMT), 2019, pp. $1-3$.

[31] C. Deng, Y. Li, Z. Zhang, and Z. Feng, "A compact broadside/conical circularly polarized antenna for pattern diversity design," in Proc. IEEE Int. Wirel. Symp. (IWS), 2014, pp. 1-3.

[32] K. Wei, Z. Zhang, W. Chen, and Z. Feng, "A novel hybrid-fed patch antenna with pattern diversity," IEEE Antennas Wireless Propag. Lett., vol. 9, pp. 562-565, 2010.

[33] S.-L. S. Yang, K.-M. Luk, H.-W. Lai, A.-A. Kishk, and K.-F. Lee, "A dual-polarized antenna with pattern diversity," IEEE Antennas Propag. Mag., vol. 50, no. 6, pp. 71-79, 2008.

[34] H. Zhong, Z. Zhang, W. Chen, Z. Feng, and M. F. Iskander, "A tripolarization antenna fed by proximity coupling and probe," IEEE Antennas Wireless Propag. Lett., vol. 8, pp. 465-467, 2009.

[35] L. Sun, W. Huang, B. Sun, Q. Sun, and J. Fan, "Two-port pattern diversity antenna for $3 \mathrm{G}$ and $4 \mathrm{G}$ MIMO indoor applications," IEEE Antennas Wireless Propag. Lett., vol. 13, pp. 1573-1576, 2014.

[36] S.-L. Yang and K.-M. Luk, "A wideband 1-probe fed patch antenna for pattern reconfiguration," in Proc. IEEE Ant. and Propag. Int. Symp., vol. 2, 2005, pp. 581-584.

[37] B. Mohamadzade, R. B. Simorangkir, S. Maric, A. Lalbakhsh, K. P. Esselle, and R. M. Hashmi, "Recent developments and state of the art in flexible and conformal reconfigurable antennas," Electronics, vol. 9, no. 9, p. 1375, 2020.

[38] R. B. V. B. Simorangkir, Y. Yang, K. P. Esselle, and B. A. Zeb, "A method to realize robust flexible electronically tunable antennas using polymer-embedded conductive fabric," IEEE Trans. Antennas Propag., vol. 66, no. 1, pp. 50-58, 2018.

[39] R. B. V. B. Simorangkir, Y. Yang, R. M. Hashmi, T. Björninen, K. P. Esselle, and L. Ukkonen, "Polydimethylsiloxane-embedded conductive fabric: Characterization and application for realization of robust passive and active flexible wearable antennas," IEEE Access, vol. 6, pp. $48102-$ $48112,2018$.

[40] A. A. Deshmukh and K. P. Ray, "Broadband proximity-fed modified rectangular microstrip antennas," IEEE Antennas Propag. Mag., vol. 53, no. 5, pp. 41-56, 2011.
[41] S. Liu, W. Wu, and D.-G. Fang, "Wideband monopole-like radiation pattern circular patch antenna with high gain and low cross-polarization," IEEE Trans. Antennas Propag., vol. 64, no. 5, pp. 2042-2045, 2016.

[42] B. Mohamadzade, R. B. V. B. Simorangkir, R. M. Hashmi, and A. Lalbakhsh, "A conformal ultrawideband antenna with monopole-like radiation patterns," IEEE Trans. Antennas Propag., 2020.

[43] B. Mohamadzade, R. B. Simorangkir, R. M. Hashmi, and K. P. Esselle, "A low profile, uwb circular patch antenna with monopole-like radiation characteristics," in 2020 International Workshop on Antenna Technology (iWAT). IEEE, 2020, pp. 1-3.

[44] D. Headland, P. Thurgood, D. Stavrevski, W. Withayachumnankul, D. Abbott, M. Bhaskaran, and S. Sriram, "Doped polymer for low-loss dielectric material in the terahertz range," Optical Materials Express, vol. 5, no. 6, pp. 1373-1380, 2015.

[45] W. A. W. Muhamad, R. Ngah, M. F. Jamlos, P. J. Soh, and M. T. Ali, "High-gain dipole antenna using polydimethylsiloxane-glass microsphere (pdms-gm) substrate for 5g applications," Applied Physics A, vol. 123, no. 1, p. 102, 2017.

[46] Y. Bayram, Y. Zhou, B. S. Shim, S. Xu, J. Zhu, N. A. Kotov, and J. L. Volakis, "E-textile conductors and polymer composites for conformal lightweight antennas," IEEE Transactions on Antennas and Propagation, vol. 58 , no. 8, pp. 2732-2736, 2010.

[47] B. Mohamadzade, R. M. Hashmi, R. B. V. B. Simorangkir, R. Gharaei, S. Ur Rehman, and Q. H. Abbasi, "Recent advances in fabrication methods for flexible antennas in wearable devices: state of the art," Sensors, vol. 19, no. 10, p. 2312, 2019.

[48] P. T. Minh, T. T. Thao, N. T. Duc, and V. Van Yem, "A novel multiband frequency reconfigurable PIFA antenna," in Proc. Int. Conf. on Adv. Tech. for Comm. (ATC), 2016, pp. 7-12.

[49] R. Garg, R. Bhartia, I. Bahl, and A. Ittipiboon, "Circular disk and ring antennas," in Microstrip Antenna Design Handbook. Boston: Artech House, Inc., 2001, ch. 5, pp. 317-398.

[50] J. Tak and J. Choi, "Circular-ring patch antenna with higher order mode for on-body communications," Micro and Opt. Tech. Letters, vol. 56, no. 7, pp. 1543-1547, 2014.

[51] B. Mohamadzade, R. B. V. B. Simorangkir, R. M. Hashmi, Y. ChaoOger, M. Zhadobov, and R. Sauleau, "A conformal band-notched ultrawideband antenna with monopole-like radiation characteristics," IEEE Antennas Wireless Propag. Lett., vol. 19, no. 1, pp. 203-207, 2020.

[52] R. B. V. B. Simorangkir, D. Le, T. Björninen, A. S. M. Sayem, M. Zhadobov, and R. Sauleau, "Washing durability of PDMS-conductive fabric composite: realizing washable UHF RFID tags," IEEE Antennas Wireless Propag. Lett., vol. 18, no. 12, pp. 2572-2576, 2019. 